\title{
Quality of Basic Care During Neonatal Resuscitation Among Health Care Providers at Three District Hospitals in Kigali
}

\author{
Kellen Muganwa $^{1 *}$, Alice Muhayimana ${ }^{1}$, Joella Mukashyaka ${ }^{1}$, Pamela Meharry² \\ ${ }^{1}$ School of Nursing and Midwifery, College of Medicine and Health Sciences, University of Rwanda, Kigali, Rwanda \\ ${ }^{2}$ Department of Women's, Children's and Family Health Services, University of Ilinois, Chicago, USA
}

*Corresponding author: Kellen Muganwa. School of Nursing and Midwifery, College of Medicine and Health Sciences, University of Rwanda, Huye Campus, Southern Province, Rwanda. Email: muganwakellen@gmail.com.

\begin{abstract}
Background

Globally, about $10 \%$ of neonates require extra respiratory support to initiate breathing at birth. Over a million neonatal deaths could be prevented with quality basic care during neonatal resuscitation.
\end{abstract}

\section{Objective}

To determine the quality of care (QoC) of basic NR at birth among health care providers (HCPs) at three district hospitals in Kigali.

\section{Methods}

A descriptive cross-sectional study was conducted. Thirty-six HCPs were observed 2-3 times using a structured checklist. Descriptive and inferential statistics were used to analyze the data.

\section{Results}

The majority had 'good' QoC scores for drying and stimulation (74.7\%), and fair scores for airway clearance $(85.1 \%)$. Some had poor scores for advanced bag and mask ventilation (BMV) (13\%). Maternity work experience (1-5 years) was significantly associated with good quality drying and stimulation $(\mathrm{p}=0.03)$, initial BMV ( $\mathrm{p}=$ 0.02), and advanced BMV ( $\mathrm{p}=0.03)$, than HCPs with less than one-year experience.

\section{Conclusion}

Maternity work experience of more than one year significantly improved the QoC during neonatal resuscitation. More NR support during the first year of work and regular NR refresher training would improve neonatal outcomes.

Rwanda J Med Health Sci 2020;3(2):225-237

\section{Keywords: Neonatal resuscitation, HBB, basic care, quality of care, health care provider}

\section{BACKGROUND}

Worldwide every year, about 2.7 million neonates die as a result of birth asphyxia and other related complications.[1] One of the most frequent causes of early death is asphyxia, which accounts for almost $23 \%$ of neonatal deaths, [2-3] and $99 \%$ of these occur in low and middle-income countries (LMICs).[1] According to the World Health Organization (WHO), about 10 million neonates do not breathe at birth, and six million require basic neonatal resuscitation (NR).[4]

Basic NR, at time of birth, comprises of a set of interventions - beginning with drying and stimulation - that is required to establish breathing and circulation in a neonate who is not spontaneously breathing or crying. [4] Approximately 10\% of all neonates require basic resuscitation at birth,[2] and less than $1 \%$ require advanced resuscitation and ongoing inpatient specialized care.[5]

The strategy to reduce neonatal mortality is to improve the quality of basic NR at birth, which is estimated to reduce the mortality rate by $30 \%$ in lowresource settings with full-term neonates.[6] Accessibility of functional equipment in all delivery areas is essential. A current review of data from six African countries, namely Ethiopia, Kenya, Madagascar, Mozambique, Rwanda, and Tanzania revealed that only $8 \%$ to $22 \%$ of sites had equipment for supporting neonatal breathing in delivery areas.[5] 
The WHO and the American Academy of Pediatrics (AAP) recommend neonatal resuscitation algorithms and essential equipment at the primary and referral level health facilities in low-resource settings. Essential NR equipment includes gloves, towels/ cloths, suction devices, ventilation devices, stethoscope, and timer.[6] A large multicenter study in Tanzania provided the initial evidence that demonstrated the effectiveness of training healthcare providers (HCP) in the Helping Babies Breathe (HBB) resuscitation program, which resulted in a $47 \%$ decrease in early neonatal mortality.[7] Perinatal conditions like birth asphyxia and hypothermia are directly linked to the quality of care at delivery and averted by using quality care to help neonates initiate and sustain breathing. $[8,9]$

Rwanda has put much effort into social and health improvements after the 1994 genocide against the Tutsi that lowered health inequities and the child mortality rate. It played a lot to achieve the Millennium Development Goal (MDG4); to decrease neonatal mortality rate as per the results from Rwanda Demographic Health Survey (RDHS) in 2015.[10] The RDHS (2014-2015) showed that the neonatal mortality rate is still quite high, at 20 deaths /100,000 live births.[11] However, challenges influence healthcare systems in many low-income countries in terms of delivering quality care and in life-saving interventions due to inadequate materials and gaps in HCP skills.[12] Strengthening HCP skills and resuscitation resources are vitally needed to achieve the quality of basic care for neonates' health and meet the Sustainable Development Goal (SDG3); to ensure healthy lives and promote well-being for all.[13]

Birth asphyxia counts as a leading cause of neonatal death in Rwanda district hospitals, with recent findings indicating a rate of $39 \% .[4,14]$ In 2012, most (94\%) neonatal deaths occurred in the early neonatal period, and of these, $68 \%$ occurred at the time of birth.[13] Quality of basic care during NR at birth could contribute to the reduction in neonatal mortality associated with asphyxia and prevent neonatal deaths, as well as improve the outcomes of neonates delivered with asphyxia. This has been demonstrated in low resource settings in Sub-Saharan Africa (SSA) and other parts of the world.[14] This study aimed to determine the quality of basic care provided during NR to contribute to evidence-based efforts to reduce neonatal deaths attributable to birth asphyxia.

\section{METHODS}

\section{Design}

The study was a descriptive, cross-sectional design and conducted in the delivery room and theatre of the maternity ward at three selected district hospitals in Kigali, Rwanda. The study sites included Masaka, Kibagabaga, Muhima due to their high neonatal mortality rate from birth asphyxia, whereby extra breathing support was needed to initiate and sustain spontaneous breathing. Data collection was conducted from February 20 to May 15, 2019.

\section{Participants' recruitment}

The non-probability method was used from the entire population of $40 \mathrm{HCPs}$ who were involved in $\mathrm{NR}$, and 95\% confidence, and p-value 0.05 were considered statistically significant. All HCPs assigned to the delivery room and maternity theatre that conducted NR, and met the inclusion criteria and voluntarily consented to participate, were recruited. Only 36 HCPs participated in the study; others were absent due to leave, sickness, or institution training. Each HCP was assessed two or three times while giving NR care to minimize the Hawthorne effect and increase the number of resuscitated neonates.

\section{Measures}

The data were collected using a structured checklist that was adapted from international guidelines, which is a valid and reliable instrument. The corresponding lead author provided permission to use the checklist via email on 13 May 2018. The investigator adopted the international checklist for the local context.[15,16] The data collection tool was arranged in seven sections:

Section 1. Social demographic characteristics (11 items) included; age, gender, $\mathrm{HCP}$ level, highest qualification level of training, NR training, prior NR training attended, duration since last NR training, support supervision in NR, staff supervision, supervisor, most recent staff supervision, maternity unit work experience, the number of HCPs and resuscitated neonates. These variables were measured as frequencies.

Section 2. Clinical protocols and guidelines (3 items) included visual NR action plans present in resuscitation areas, HCPs use NR action plans, and guidelines during resuscitation, flip charts of NR provided in the labor ward. Items were scored with "Yes" (1) or "No" (0) and presented as frequencies. 
Section 3. Basic NR equipment in the labor ward and maternity theatre were assessed for permanent equipment (items 5) and temporary equipment (items 6) scored "present" (1) or "absent" (0), and presented as frequencies.

Section 4. Skills of basic care via four principal steps in NR include; drying and stimulation, airway clearance, initial BMV, and advanced or improved BMV (4 items). The variables were measured in frequencies and categorized "poor" (missed all or majority items in recommended steps), "fair" (half the recommended steps performed), "good" (performed all or majority items in recommended steps).[15]

Section 5. NR's outcome variables on improving quality care included the response of neonates to drying and stimulation, clearing the airway, initial BMV, assisted/ improved BMV, and assisted/ support ventilation (5 items). Items were scored with "Yes" (1) or "No" (0) and presented as frequencies.

Section 6. Infection prevention practices provided by HCPs included cleaning and disassembled device while wearing gloves, decontamination in chloride solution $5 \%$ for $10-20$ minutes, all parts washed with soap and water and rinsed with clean water, parts dried completely (4items). Items were measured as "Yes" (1) or "No" (0) and presented as frequencies.

Section 7. A Logistic regression model was used to identify statistical associations between HCPs' characteristics and the QoC during NR.

\section{Data collection}

The arrangement of data collection was made by selecting three data collector assistants (RAs) in the postnatal unit of the maternity ward in each selected hospital. The RAs were trained on the objectives, the benefit of the study, the individual's rights, informed consent, and how the structured checklist tool was used. The investigator selected staff members from other services, especially those with experience in postnatal and NR training, to be RAs. Staff members were explicitly selected to observe and assess the HCP two or three times while giving NR care, and familiarity of the observers minimized the likelihood of the Hawthorne effect. The assumption was that HCPs would be less likely to change their practices when observed by someone familiar in the same unit than an observer from outside the hospital. The observer followed the delivery that came first. The investigator and RAs endeavored to stand next to the resuscitation table, without interfering in the NR process.

Most of the HCPs were approached individually by the RAs due to the different work shifts. However, consented HCPs/participants were recruited and requested to complete the structured observation checklist on the social demographic data, including information on training and qualifications, work experience, and refresher training attended. The participants signed the written consent and agreed to be observed during NR by the RA, and therefore no subsequent reminders were necessary during the actual procedure.

During the first week of data collection at each site, the investigator worked closely with RAs during the day and evening hours to ensure that they could complete the direct observation structured checklist consistently and correct any mistakes at the end of each day. The investigator would receive the report from each RA. The data collection took ten weeks to complete.

\section{Data analysis}

The variables were coded and entered into SPSS Statistics (version 21). The data were analyzed using descriptive and inferential statistics. Frequencies were calculated for the demographic variables. Logistic regression used the Beta level to assess the effect of demographic characteristics on NR quality. Results with a p-value $<0.05$ were considered statistically significant.

\section{Ethical considerations}

Approval was obtained from the University of Rwanda, College of Medicine and Health Science Institutional Review Board, and three study sites. All participants signed the consent form prior to data collection.

\section{RESULTS}

This cross-sectional design aimed to determine the quality of basic care provided during NR in the delivery room and theatre of the maternity ward in three district hospitals. Data collection was conducted from February 20 to May 15, 2019. 


\section{Sociodemographic characteristics of participants}

Table 1. Sociodemographic characteristics of participants

\begin{tabular}{|c|c|c|}
\hline $\begin{array}{l}\text { Healthcare Providers (HCP) } \\
\text { Characteristics }\end{array}$ & HCPs (n=36) & $\begin{array}{l}\text { Neonates }(n=87) \\
\text { Resuscitated }\end{array}$ \\
\hline \multicolumn{3}{|l|}{ Age (years) } \\
\hline$>25$ & $4(11.1)$ & $9(10.3)$ \\
\hline$\geq 25$ & $32(88.9)$ & $78(89.7)$ \\
\hline \multicolumn{3}{|l|}{ Gender } \\
\hline Male & $15(41.7)$ & $38(43.7)$ \\
\hline Female & $21(58.3)$ & 49 (56.3) \\
\hline \multicolumn{3}{|l|}{ HCP level } \\
\hline Midwife/ Nurse & $34(94.4)$ & $82(94.3)$ \\
\hline Medical doctor & $1(2.8)$ & $4(4.6)$ \\
\hline Specialist (Obs /gyn) & $1(2.8)$ & $1(1.21)$ \\
\hline \multicolumn{3}{|l|}{ Highest training qualification } \\
\hline Diploma & $27(75.0)$ & $77(88.5)$ \\
\hline Bachelor's degree & $8(22.2)$ & $9(10.4)$ \\
\hline Master's degree & $1(2.8)$ & $1(1.1)$ \\
\hline \multicolumn{3}{|l|}{ Training in NR } \\
\hline Yes & $31(86.1)$ & $76(87.4)$ \\
\hline No & $5(13.9)$ & $11(12.6)$ \\
\hline Prior NR training attended $(n=31)$ & & $\mathbf{n}=76$ \\
\hline Helping Babies Breathe, Yes & $30(96.6)$ & $43(56.6)$ \\
\hline Helping Babies Breathe, No & $1(3.2)$ & $33(43.4)$ \\
\hline \multicolumn{3}{|l|}{ Pediatric Advanced Life Support } \\
\hline Yes & $16(51.6)$ & $70(18.4)$ \\
\hline No & $15(48.4)$ & $6(92.1$ \\
\hline \multicolumn{3}{|l|}{ Emergency Triage Assess. \& Treatment } \\
\hline Yes & $14(45.1)$ & $35(46.1)$ \\
\hline No & $17(54.8)$ & $41(53.9)$ \\
\hline \multicolumn{3}{|c|}{ Soins Obstetricauxet neonatal d'urgence } \\
\hline Yes & $1(3.2)$ & $50(70.1)$ \\
\hline No & $30(96.8)$ & $26(26.2)$ \\
\hline Duration since last NR training & & $n=61$ \\
\hline$<6$ months & $15(48.4)$ & $20(32.7)$ \\
\hline$\geq 12$ months & $16(51.6)$ & $41(67.3)$ \\
\hline \multicolumn{3}{|l|}{ Support supervision in NR } \\
\hline Yes & $18(63.9)$ & $38(78.6)$ \\
\hline No & $13(36.1)$ & $23(21.4)$ \\
\hline \multicolumn{3}{|l|}{ Staff supervision $(n=23)$} \\
\hline$<6$ months & $3(13.0)$ & $7(11.5)$ \\
\hline 6-12 months & $5(21.8)$ & $12(19.6)$ \\
\hline$\geq 12$ months & $15(65.2)$ & $42(68.9)$ \\
\hline \multicolumn{3}{|l|}{ Supervisor } \\
\hline Manager & $11(48.0)$ & $27(44.3)$ \\
\hline Maternal Child Survival Programme & $6(26.0)$ & $15(24.5)$ \\
\hline
\end{tabular}




\begin{tabular}{lcc}
\hline Ward mate/ colleague & $6(26.0)$ & $19(31.2)$ \\
Practice in maternity unit (years) & $9(13.9)$ & $13(11.4)$ \\
$<1$ & $12(83.3)$ & $40(80.4)$ \\
$>1-5$ & $2(2.8)$ & $8(9.2)$ \\
$\geq 5$
\end{tabular}

HCPs: Health Care Providers, NR: Neonatal Resuscitation, HBB: Helping Babies Breathe, PALS: Pediatric Advanced Life Support, ETAT: Emergency Triage Assessment and Treatment, SONU: SoinsObstetricauxet neonatal d'urgence, MCSP: Maternal Child Survival Programme

Table 1. Shows the participant's Socio-demographic characteristics. The majority (58.3\%) was female, older than 25 years $(88.9 \%)$, a midwife or nurse $(94.4 \%)$, and registered advanced diploma holder $(75.0 \%)$. The majority $(86.1 \%)$ reported being trained in NR, and most $(96.6 \%)$ were trained in the Helping Babies Breathe (HBB) program. The majority (51.6\%) had NR training over 12 months ago, had NR supervision (63.9\%), and staff supervision was greater than 12 months ago (65.2\%). The majority (48.0\%) reported the unit manager was the supervisor. The majority (83.3\%) of HCPs reported one to five years maternity work experience.

\section{Availability of the basic equipment in neonatal resuscitation at birth}

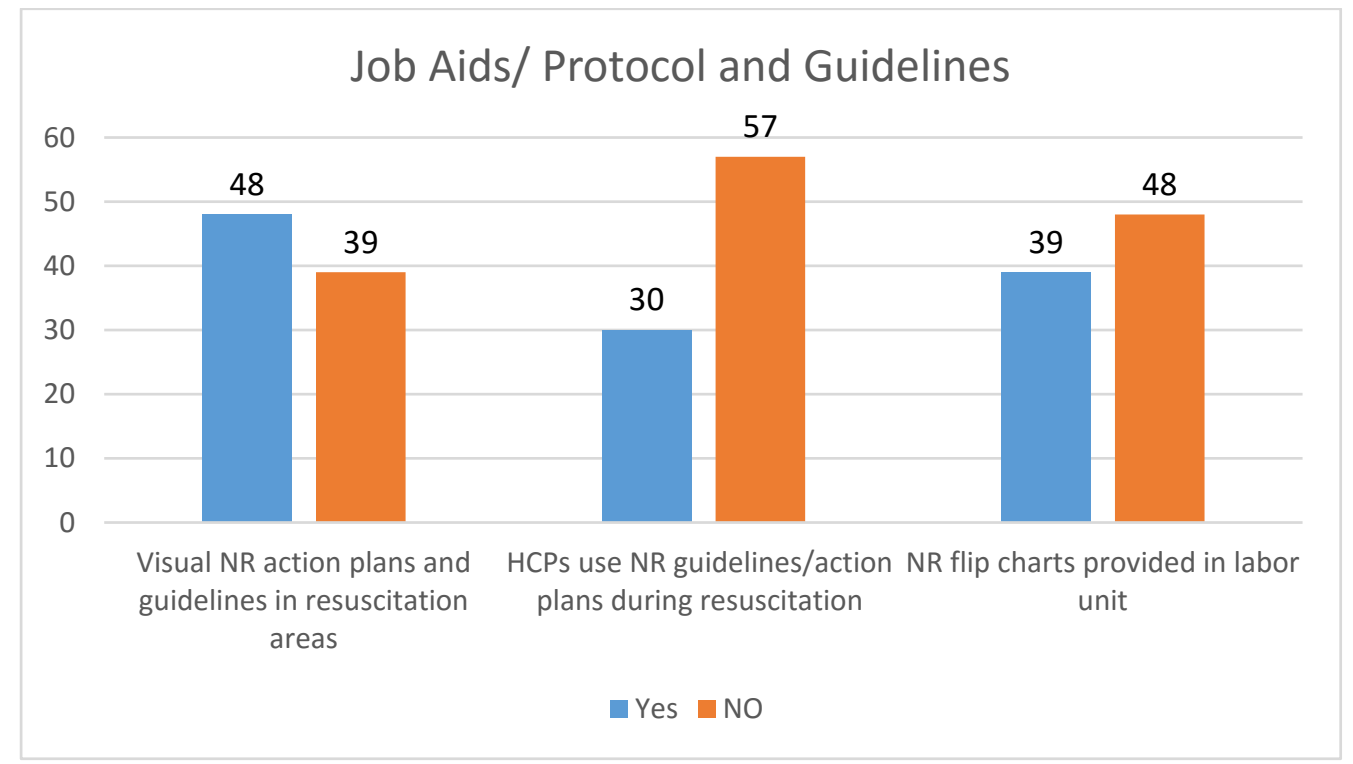

Figure 1 Availability of basic equipment in neonatal resuscitation at birth

Figure 1 shows the availability of the basic equipment in NR at birth. During resuscitation, over half (55.2\%) of Visual NR action plans/guidelines were present in the resuscitation areas. However, during resuscitation, the majority $(65.5 \%)$ did not refer to NR guidelines/action plans. Over half $(55.2 \%)$ of NR flip charts for neonatal resuscitation were not provided in the labor unit. 


\section{Basic equipment available in NR at birth}

Table 2. Basic neonatal resuscitation equipment at birth

\begin{tabular}{|c|c|c|c|c|}
\hline \multirow[t]{2}{*}{ Equipment } & \multicolumn{2}{|c|}{ Labor Ward n (\%) } & \multicolumn{2}{|c|}{ *Theatre $\mathbf{n}(\%)$} \\
\hline & Present & Absent & Present & Absent \\
\hline \multicolumn{5}{|l|}{ Permanent Items } \\
\hline Warmer/ resuscitative & $65(89.0)$ & $8(11.0)$ & $13(92.9)$ & $1(7.1)$ \\
\hline Oxygen source & $53(72.6)$ & $20(27.4)$ & $12(85.7)$ & $2(14.3)$ \\
\hline Suction machine & $59(80.8)$ & $14(19.2)$ & $11(78.6)$ & $3(21.4)$ \\
\hline Ambu bag (500 mls) & $46(63.0)$ & $27(37.0)$ & $12(85.7)$ & $2(14.3)$ \\
\hline Clock & $3(4.1)$ & $70(95.9)$ & $0(0)$ & $14(100)$ \\
\hline \multicolumn{5}{|l|}{ Temporary Items } \\
\hline Suction tube $(6 \mathrm{~F}, 8 \mathrm{~F}, 10 \mathrm{~F})$ & $49(67.1)$ & $24(32.2)$ & $11(78.6)$ & $3(21.4)$ \\
\hline Bulb suction device (Penguin) & $50(68.5)$ & $23(31.5)$ & $13(92.9)$ & $1(7.1)$ \\
\hline Face mask (preterm ' 0 ', term ' 1 ') & $17(23.3)$ & $56(76.7)$ & $10(71.4)$ & $4(28.6)$ \\
\hline Oxygen delivery devices ${ }^{* *}$ & $45(61.6)$ & $28(38.4)$ & $12(85.7)$ & $2(14.3)$ \\
\hline Oxygen tubing & $44(60.3)$ & $29(39.7)$ & $14(100.0)$ & $0(0.0)$ \\
\hline Clean, dry towels (2) & $36(49.3)$ & $37(50.7)$ & $12(85.7)$ & $2(14.3)$ \\
\hline
\end{tabular}

*Maternity Theatre ${ }^{* *}$ Oxygen delivery devices (nasal catheter, prongs, face mask)

Table2.Shows the presence of permanent and temporary items in the labor ward or maternity theatre at the time of birth. The following permanent items were present in the labor ward; warmer/resuscitative $(89.0 \%)$, oxygen source $(72.6 \%)$, suction machine $(80.8 \%)$, Ambu bag (500mls) and $(63.0 \%)$. The clock was absent for resuscitations most of the time (95.9\%). Permanent items present in the maternity theatre included; warmer/ resuscitative $(92.9 \%)$, oxygen source $(85.7 \%)$, suction machine $(78.6 \%)$, and Ambu bag (500mls) $(85.7 \%)$. The clock was absent in all resuscitations (100.0\%).
The following temporary items were available for resuscitation. Temporary items present in the labor ward included; suction tube $(6 \mathrm{~F}, 8 \mathrm{~F}$, and $10 \mathrm{~F})$ $(67.1 \%)$, bulb suction device (Penguin device, colored bulb sucker) (68.5\%), face mask (preterm: size 0; term: size 1) (23.3\%), oxygen delivery device (nasal catheter, nasal prongs, face mask) (61.6\%), oxygen tubing $(60.3 \%)$, and clean, dry towels $(49.3 \%)$. Temporary items present in the maternity theatre included; suction tube $(6 \mathrm{~F}, 8 \mathrm{~F}$, and $10 \mathrm{~F})$ (78.6\%), bulb suction device (Penguin device, colored bulb sucker) $(92.9 \%)$, face mask (preterm: size 0; term: size 1) (71.4\%) oxygen delivery device (nasal catheter, nasal prongs, face mask) (85.7\%), oxygen tubing $(100.0 \%)$, and clean, dry towels $(85.7 \%)$. 


\section{Quality of care scores of the four principles steps in neonatal resuscitation}

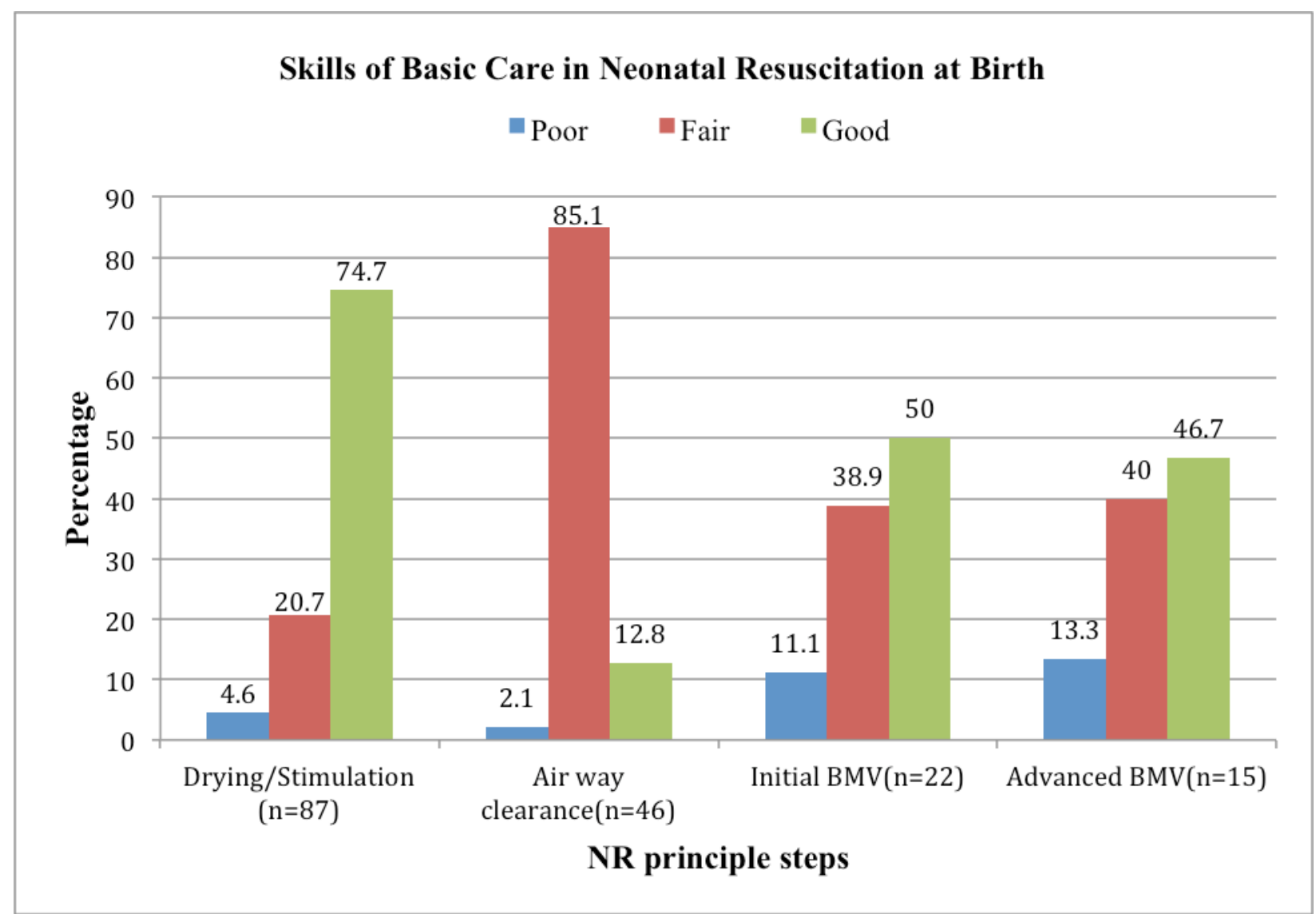

\section{Figure 2. Basic care skill in neonatal resuscitation at birth}

Figure 2. Shows the majority of HCPs had 'good' drying and stimulation skills (74.7\%), initial BMV skills (50\%), and advanced BMV skills (46.7\%). The majority (85.1\%) of HCPs provided only 'fair' airway clearance, and only a limited number (12.8\%) provided 'good' care. 


\section{Outcomes of neonatal resuscitation in providing quality care}

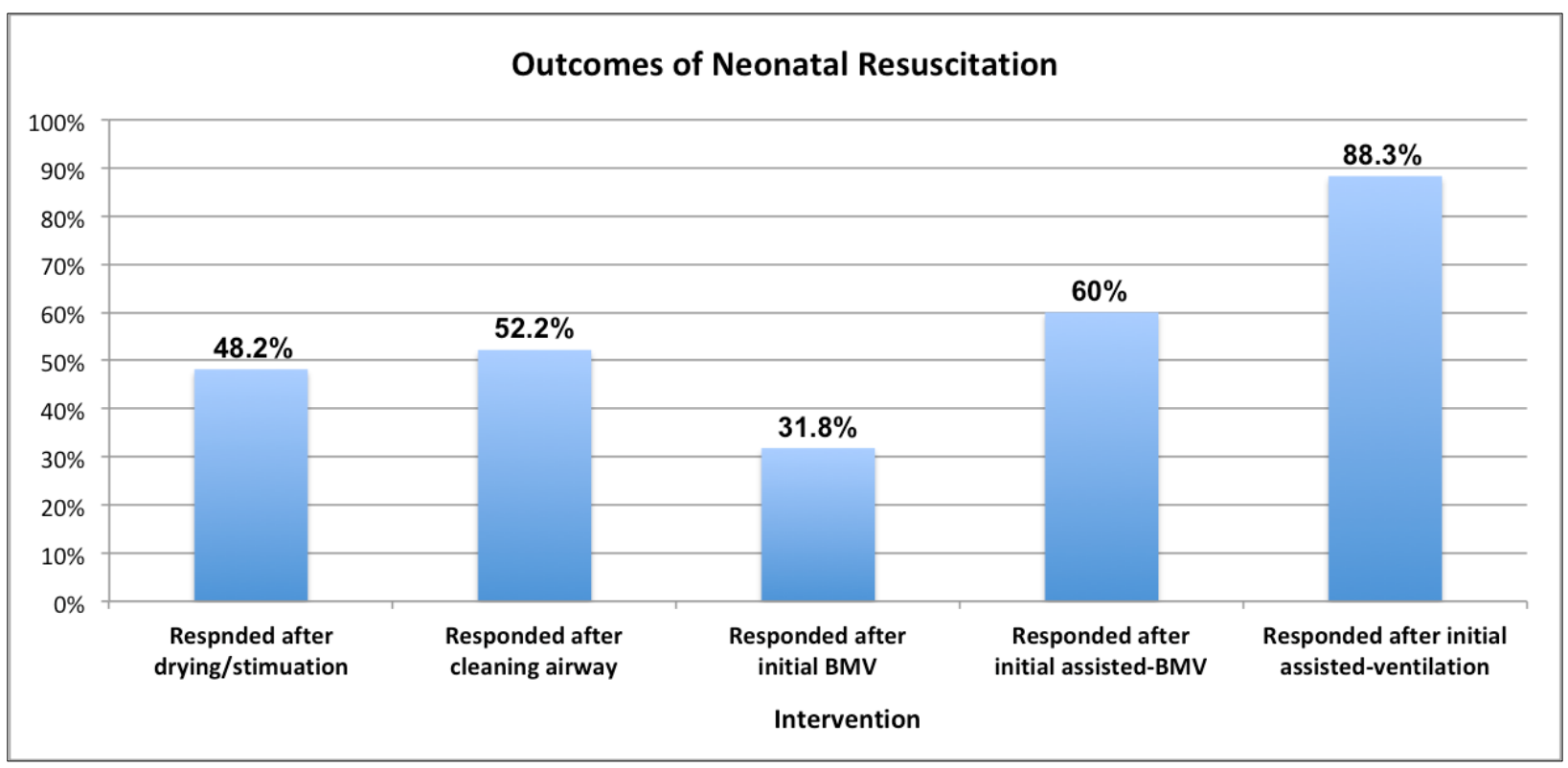

Figure 3. Outcomes of neonatal resuscitation in providing quality care

Figure 3. Shows that among the resuscitated neonates, nearly half $(48.2 \%)$ responded to drying / stimulation and among those who did not respond to drying/stimulation, the majority $(52.2 \%)$ responded to airway clearance. Among the neonates who did not respond to airway clearance, nearly a third (31.8\%) responded to initial BMV, and of those who did not respond to initial BMV, the majority $(60 \%)$ responded to assisted BMV. Among those that did not respond to assisted BMV, the majority $(88.3 \%)$ responded to support or improved ventilation. None of the observed neonates died.

\section{Infection prevention practices}

\section{Table 3. Infection prevention practices}

\begin{tabular}{lcc}
\hline Prevention Practices & Yes n (\%) & No n (\%) \\
\hline $\begin{array}{l}\text { - Cleaning - ventilation bag and mask device disassembled } \\
\text { (suction device if possible) while still wearing gloves. }\end{array}$ & $22(25.3)$ & $65(74.7)$ \\
$\begin{array}{l}\text {-Decontamination - all parts soaked in a 5\% chlo-ride/solution } \\
\text { for 10-20 minutes. }\end{array}$ & $3(3.4)$ & $84(96.6)$ \\
$\begin{array}{l}\text {-All parts washed with soap and water and rinsed carefully with } \\
\text { clean water to remove all soap. }\end{array}$ & $42(48.3)$ & $45(51.7)$ \\
-Dry entirely and keep clean until next use & $43(49.4)$ & $44(50.6)$
\end{tabular}

The findings show that three quarters $(74.7 \%)$ did not disassemble and clean the NR equipment, e.g., the bag and mask device was disassembled, while still with gloves on was not observed (Table 3). Furthermore, nearly all (96.6\%) HCPs did not decontaminate the equipment by soaking all the parts in a $5 \%$ chloride solution for 10 to 20 minutes. The majority also did not rinse the parts thoroughly $(51.7 \%)$, or dry completely and keep in a clean place for the next NR (50.6\%). 
Logistic regression of QoC scores with HCPs' characteristics.

Table 4. Logistic regression of the QoC scores with HCPs' characteristics

\begin{tabular}{|c|c|c|c|}
\hline HCP Characteristics & $\beta$ & $95 \% \mathrm{CI}$ & $\mathbf{p}$ \\
\hline \multicolumn{4}{|c|}{${ }^{*}$ Drying and stimulation professional training } \\
\hline Midwives and Nurses & 0.45 & $0.124-1.433$ & 0.33 \\
\hline \multicolumn{4}{|l|}{ NR training } \\
\hline \multicolumn{4}{|l|}{ No (ref) } \\
\hline Yes & 0.32 & $0.101-3.82$ & 0.21 \\
\hline \multicolumn{4}{|l|}{ Maternity experience } \\
\hline $1-5$ years & 1.76 & $1.23-2.93$ & 0.03 \\
\hline$>5$ years & 1.97 & $1.83-3.87$ & 0.01 \\
\hline \multicolumn{4}{|l|}{ Supervision Support } \\
\hline Yes & 1.76 & $1.65-2.31$ & 0.21 \\
\hline \multicolumn{4}{|c|}{ *Airway maintenance professional training } \\
\hline \multicolumn{4}{|l|}{ Medical doctors (ref) } \\
\hline \multirow{2}{*}{\multicolumn{4}{|c|}{ NR training }} \\
\hline & & & \\
\hline \multicolumn{4}{|l|}{ No (ref) } \\
\hline \multirow{2}{*}{\multicolumn{4}{|c|}{ Maternity work experience }} \\
\hline & & & \\
\hline $1-5$ years & 1.46 & $1.13-2.13$ & 0.04 \\
\hline$>5$ years & 1.57 & $1.33-2.87$ & 0.03 \\
\hline \multicolumn{4}{|l|}{ Supervision Support } \\
\hline Yes & 1.16 & $1.25-2.71$ & 0.41 \\
\hline \multicolumn{4}{|c|}{ * Initial BMV professional training } \\
\hline \multicolumn{4}{|l|}{ Medical doctors (ref) } \\
\hline Midwives and Nurses & 0.65 & $0.32-1.93$ & 0.43 \\
\hline \multicolumn{4}{|l|}{ NR training } \\
\hline \multicolumn{4}{|l|}{ No (ref) } \\
\hline Yes & 0.72 & $0.12-2.82$ & 0.11 \\
\hline \multicolumn{4}{|c|}{ Maternity work experience } \\
\hline \multicolumn{4}{|c|}{$<1$ year (ref) } \\
\hline $1-5$ years & 1.66 & $1.73-2.97$ & 0.02 \\
\hline$>5$ years & 1.87 & $1.43-3.13$ & 0.02 \\
\hline \multicolumn{4}{|l|}{ Supervision Support } \\
\hline \multicolumn{4}{|l|}{ No (ref) } \\
\hline Yes & 1.76 & $1.65-2.31$ & 0.21 \\
\hline $\begin{array}{l}\text { *Advanced BMV prof } \\
\text { Medical doctors (ref) }\end{array}$ & & & \\
\hline Midwife / Nurses & 0.85 & $0.174-2.43$ & 0.13 \\
\hline NR training & & & \\
\hline No (ref) & & & \\
\hline Yes & 0.92 & $0.801-2.12$ & 0.31 \\
\hline Maternity work expe & & & \\
\hline <1 year (ref) & & & \\
\hline $1-5$ years & 1.66 & $1.53-2.13$ & 0.03 \\
\hline$>5$ years & 2.07 & $1.71-3.47$ & 0.02 \\
\hline Supervision Support & & & \\
\hline No (ref) & & & \\
\hline Yes & 1.86 & $1.85-2.41$ & 0.20 \\
\hline
\end{tabular}

QoC: Quality of care, HCP: Health Care Providers, NR: Neonatal Resuscitation, BMV: Bag and Mask Ventilation 
Table4. Logistic regression was used to analyze the effect of demographic characteristics on the quality of NR presented in table 1 . The following results include drying and stimulation, Airway maintenance, Initial BMV, and Advanced BMV.

\section{Drying and stimulation}

The results revealed that HCPs who reported having 1-5 years maternity work experience were more likely to provide good quality drying/stimulation $(\beta=$ $1.76, \mathrm{CI}=1.23-2.93, \mathrm{p}=0.03)$, and those with more than five years experience were nearly two times more likely to provide good quality drying/stimulation $(\beta=1.97, \mathrm{CI}=1.83-3.87, \mathrm{p}=$ $0.01)$ than those with one-year experience.

\section{Airway maintenance}

HCPs with 1-5 years of experience in maternity were more likely to provide good quality airway maintenance $(\beta=1.46, C I=1.13-2.13, p=0.04)$ than those with one year, and those with more than five years were more likely to provide good quality airway maintenance $(\beta=1.57, \mathrm{CI}=1.33-2.87, \mathrm{p}=$ $0.03)$ than those with one-year experience.

\section{Initial BMV}

HCPs with 1-5 years of experience in maternity were more likely to provide good quality initial BMV ( $\beta=$ $1.66, \mathrm{CI}=1.73-2.97, \mathrm{p}=0.02$ ) than those with one year, and those with more than five years' experience were more likely to provide good quality initial BMV $(\beta=1.87, \mathrm{CI}=1.43-3.13, \mathrm{p}=0.02)$ than those with one year experience.

\section{Advanced BMV}

HCPs who reported having 1-5 years of experience in maternity were more likely to provide good quality advanced BMV $(\beta=1.66, \mathrm{CI}=1.53-2.13, \mathrm{p}=0.03)$ than those with one year, while those with more than five years' experience were more than two times more likely to provide good quality advanced BMV $(\beta=2.07, \mathrm{CI}=1.71-3.47, \mathrm{p}=0.02)$ than those with one year experience. Other variables that were not statistically significant included professional training, NR training, and supervisory support.

\section{DISCUSSION}

This study aimed to determine the quality of basic care during NR by 36 HCPs in three selected district hospitals in Kigali City. Our study has shown that the majority of HCPs (88.9\%) were older than 25 years, female $(58.3 \%)$, and nurses and midwives $(94.4 \%)$, had been trained in NR $(86.1 \%)$, and had more than one - year maternity work experience $(86.1 \%)$.Thus, this is similar to the study done by de Graft-Johnson and colleagues of six SSA countries, including Rwanda, showed that $81.6 \%$ of HCPs were female, and $85.1 \%$ nurses and midwives.[5]

\section{Health care providers' skills}

The majority $(86.1 \%)$ of $\mathrm{HCPs}$ had received NR training, and nearly all $(96.8 \%)$ reported being trained in the HBB program. Therefore, our findings are similar to the study conducted in a low resource setting; a systemic literature review conducted in LMIC found that NR training can significantly increase HCPs skills. [7,10,17]

Midwives and Nurses provided NR to the majority of neonates $(94.2 \%)$ than other HCP $(5.8 \%)$, similar to a study conducted in Zambia on NR skills in HCP. $[2,18]$ Our findings also showed that HCPs with more than one-year maternity unit experience $(76.9 \%)$ did more resuscitation than those with less than one year experience $(21.3 \%)$. These findings were similar to other studies identified by WHO indicated that a more extended period of working on the same unit enhances the HCPs' clinical practice, self-efficacy, and competency in NR skills.[19]

The majority of HCPs had 'good' drying and stimulation skills $(74.7 \%)$, initial BMV skills (50\%), and advanced BMV skills (46.7\%). However, the majority $(85.1 \%)$ had a 'fair' score for basic skills in airway clearance, and only a limited number $(12.8 \%)$ provided 'good' care. The basic care skills with the highest 'poor' score were advanced BMV $(13.3 \%)$. A study conducted in Kenya reported that the overall QoC scores were good for airway clearance $(83 \%)$, though steps were poorly performed during drying, airway maintenance in meconium presence, and ventilation.[15]

In addition, supportive supervision was inadequate for more than half during their practice after receiving NR training, which is similar to another study conducted in Rwanda, where HCPs reported that they did not receive supervision.[20] A systematic review and a multi-country analysis emphasized supervisions and in-service training to identify the performance gaps and weaknesses.[2122]

Our findings revealed that maternity experience is associated to QoC, therefore other studies that reported maternity work experience of more than one year was statistically significant in providing quality of care during NR.[15,16,19] Variables other than maternity work experience were not statistically significant and included professional training, NR training, and supervisory support. 
Besides this, the completion of NR training does not imply that an individual is competent to perform NR, as demonstrated by the American Heart Association (AHA) neonatal resuscitation program (NRP).[19] However, it has been demonstrated elsewhere that training courses in NR can effectively increase the skills of HCPs in conducting NR and reducing potentially harmful practices.[16] There is evidence that NR training alone may not be enough to ensure a change in practice and retention of skills, but may need to be followed up with regular refresher training, as frequently as every six months to prevent loss of acquired skills.[23]

\section{Availability of equipment during Neonatal Resuscitation}

Our findings showed the availability of most NR equipment was reasonably good in the labor ward, with the presence of a warmer $(89.0 \%)$, oxygen source $(72.6 \%)$, a suction device $(80.8 \%)$, and Ambu bags $(63.0 \%)$ in the resuscitation area. These findings were similar to a study conducted in Kenya and the six sub-Saharan African countries. [5,15] According to the study conducted by De Graft Johnson et al.,(2017) reported that most facilities had some supplies of NR equipment, from a high $90.7 \%$ availability of suction devices and $81.9-100 \%$ for bag and mask to gaps in basics, such as $40.5 \%$ for towels and blankets essential for thermoregulation. $[5,24]$ The availability of NR equipment varied depending on the type of health facility; it was typically higher in hospitals, and lower in health centers.

Furthermore, our findings revealed low or no availability of other basic requirements recommended by WHO or the HBB list, including a clock and clothes. In our study, clean and dry towels were present half the time on the labor ward $(49.3 \%)$, similar to $40.5 \%$ recorded to the presence of basic requirements in the study done in SSA study.[5] Considering the importance of recording time during NR, it was surprising not to find a clock in the majority of delivery rooms (95.7\%) and maternity theatres (100.0\%). The absence of clocks was similar to a study conducted in Zambia.[2] But in contrast to a survey in Vietnam, whereby $50.3 \%$ of the 187 health facilities had clocks.[24]

Protocols and guidelines were present for NR, with over half $(55.2 \%)$ having visual NR action plans and guidelines, and NR flip charts (55.2\%) available on the labor and maternity theater units. Though the majority $(65.5 \%)$ of HCPs did not refer to them during NR intervention. In contrast, research conducted in Kenya and 12 countries in the African and Asian continents showed poor adherence to guidelines and protocols, and non-implementation of quality improvement activities.[8,19] Furthermore, the researchers mentioned that many LMIC lacked appropriate policies and guidelines for providing quality health care to neonates.[8] The presence of visual NR action plans, guidelines, and NR flip charts on the labor ward increases the chance for HCP to perform standardized NR procedure to neonates with asphyxia. We believe these findings are useful for guiding an HCP to prepare equipment and ensure that all birthing facilities in the district hospitals have essential supplies.

\section{Outcomes of basic NR in providing quality of care}

Among the neonates who required NR, almost half $(48.2 \%)$ responded to drying and stimulation, and if they did not, over half $(52.2 \%)$ responded to airway clearance and oxygen support. No neonates died. In contrast, the study in six SSA countries reported $89 \%$ of neonates either recovered on their own, or through initial stimulation by the HCP, or possibly ventilation, and $11 \%$ of neonates died.[5] Nearly half of those who required NR were not dried immediately, which increased the risk of hypothermia and less likelihood of response to resuscitation efforts.[5] A large multicenter study in Tanzania demonstrated that stimulation skills increased from $47 \%$ (before) to $88 \%$ (after) implementation of an HBB training session.[7] In contrast, the use of facemask ventilation decreased from $8.2 \%$ to $5.2 \%$, and early neonatal mortality decreased from 13.4 to 7.1 per 1000 live-born births post-HBB training in Tanzania.[7]

\section{Infection prevention}

The findings showed that three quarters $(74.7 \%)$ did not clean the NR equipment e.g. the bag and mask devices were assembled while still wearing gloves. Furthermore, nearly all $(96.6 \%)$ HCPs did not respect the decontamination process, whereby all parts should be soaked in a $5 \%$ chloride solution for duration of 10 to 20 minutes. The same line with a study conducted in Kenya indicated no high-level disinfection was done on NR equipment.[15]

\section{Limitations}

This study was conducted in three district hospitals in Kigali, Rwanda, with a non- probability sampling of HCPs who provided NR at the time of birth. The data were based on the quality of basic care during NR among the HCPs at birth. There was a risk of the observer feeling obliged to assist during the resuscitation phase if the neonate was not well treated, but that did not happen. The HCPs knew why the researchers were there, so to reduce potential bias; the RAs were recruited from other services, and fully aware of study procedures and 
supervision. The sample size was small; therefore, these findings cannot be generalized to other populations. Thus the district hospitals were limited due to the smaller number of births, and many of HCPs were absent.

\section{Recommendations}

The HCPs working in the maternity delivery unit and the theatre need more educational opportunities to learn and practice NR, and adequate equipment so they can carry procedures. According to our findings, quality basic care during NR is highly needed to reduce neonatal morbidity and mortality around the time of birth. Furthermore, a focus is needed on the preparation of NR equipment before birth and cleaning the equipment after birth. The WHO recommendation is to prepare the NR equipment and check for proper functioning before conducting a delivery.[25] In the study, the check of NR equipment availability was at $32.2 \%$ and $40.2 \%$ checks their functioning. A written checklist (laminated) for equipment and material preparation could be used for daily checks so that supplies are reordered and replenished routinely.[24,26]

Further research is highly needed to improve the quality of care in NR to reduce the high numbers of birth asphyxia and improve neonatal outcomes. Future studies should have a larger sample size and additional study sites and include quantitative and qualitative data.

\section{CONCLUSION}

This study's findings show that maternity work experience of more than one year was statistically significant in providing quality care during NR, hence reducing the risk of asphyxia and other adverse outcomes. Health facilities need to be equipped to save neonates at risk at birth. Delays in receiving quality basic care in routine life-saving situations in low-resource settings is concerning. More effort is needed to provide quality newborn health interventions, particularly during NR to overcome critical gaps, and improve the likelihood of meeting the SDG3 by 2030 or earlier.

\section{Authors' contributions}

$\mathrm{KM}$ contributed to the original work of the study, data collection, data analysis and manuscript writing. JM revised the manuscript's paper; PM and AM supervised the research study, all contributed to the writing and approved the work to be submitted.

\section{Conflict of interest declaration}

All authors declare that they have no competing financial or any other interest in relation to the work.

\section{Acknowledgments}

We are grateful to the data analysis facilitator, healthcare providers and research assistants from the three district hospitals in Kigali who made this study possible.

This article is published open access under the Creative Commons Attribution-NonCommercial NoDerivatives (CC BYNCND4.0). People can copy and redistribute the article only for noncommercial purposes and as long as they give appropriate credit to the authors. They cannot distribute any modified material obtained by remixing, transforming or building upon this article. See https://creativecommons.org/licenses/by-ncnd $/ 4.0 /$

\section{REFERENCES}

1. Aguirre LC, Vaz LME, Singh K, Sitrin D, Moran $\mathrm{AC}$, Khan SM, et al. Measuring coverage of essential maternal and newborn care interventions: An unfinished agenda. J Glob Health[internet].2017;7(2):1-5. Available from: https//www.healthnewbornnetwork.org/hnncontent/uploads/JoGH-MeasurementSupplement-Editor-Dec-2017.pdf

2. Mistry SC, Lin R, Mumphansha H, Anesthesia M, Kettley LC, Pearson JA, et al. Newborn Resuscitation Skills in Health Care Providers at a Zambian Tertiary Center, and Comparison to World Health Organization Standards. 2018;127(1):217-23.

3. Dempsey E, Pammi M, Ac R, Kj B. Standardised formal resuscitation training programmes for reducing mortality and morbidity in newborn infants ( Review ). cochranelibrar [internet]. 2015;(9). Available from: https://pubmed.ncbi,nlm.nih.gov/26337958/

4. Uwingabire F, Gowan M. Birth Asphyxia at a District Hospital in Kigali , Rwanda. Rwanda J Med Health Sci. 2019;2(2):96-104.

5. De Graft-Johnson J, Vesel L, Rosen HE, Rawlins B, Abwao S, Mazia G, et al. Crosssectional observational assessment of quality of newborn care immediately after birth in health facilities across six sub-Saharan African countries. BMJ Open. 2017;7(3). avaiable from: https://pubmed.ncbi.nlm.nih.gov/28348194/

6. Berhe AK, Tinsae F, Gebreegziabher G. Knowledge and practice of immediate newborn care among health care providers in eastern zone public health facilities.BMC Pediatrics. 2017;1-9.

7. Msemo AG, Massawe A. Newborn Mortality and Fresh Stillbirth Rates in Tanzania After Helping Babies Breathe Training. Pediatrics. 2019;131(2). 
8. Enweronu-laryea C, Dickson KE, Moxon SG, Simen-kapeu A, Nyange C, Niermeyer S, et al. Basic newborn care and neonatal resuscitation : a multi-country analysis of health system bottlenecks and potential solutions. BMC Pregnancy Childbirth [Internet]. 2015;15(Supp1 2):S4. Available from: http: / / www.biomedcentral.com/1471$2393 / 15 / \mathrm{S} 2 / \mathrm{S} 4$

9. Chikuse B, Chirwa E, Maluwa A, Malata A, Odland J. Midwives ' adherence to guidelines on the management of birth asphyxia in Malawi. Open J Nurs. 2012;2(December):3517.Available from: https

10. Musafili A. Child survival in Rwanda: Challenges and potential for improvement. $\mathrm{PhD}$ Dissertation, Uppsala University . 2015.

11. NISR. Mortality Assessment Survey Mortality Assessment Survey. 2015.

12. Hategeka C, Shoveller J, Tuyisenge L, Kenyon C, Cechetto F, Lynd LD. Pediatric emergency care capacity in a low- resource setting: An assessment of district hospitals in Rwanda. $2017 ; 1-13$.

13. SDGs. The Sustainable Development Goals Report. 2017;

14. Khurmi MS, Sayinzoga F, Berhe A. Newborn Survival Case Study in Rwanda - Bottleneck Analysis and Projections in Key Maternal and Child Mortality Rates Using Lives Saved Tool ( LiST ). Int J MCH AIDS. 2017;6(2):93-108.

15. Shikuku DN, Milimo B, Ayebare E, Gisore P, Nalwadda G. Quality of Care during Neonatal Resuscitation in Kakamega County General Hospital, Kenya: A Direct Observation Study. Biomed Res Int [Internet]. 2017;2017:1-12. Available from: https://www.hindawi.com/journals/bmri/201 $7 / 2152487 /$

16. Kamath-rayne BD, Thukral A, Visick MK, Schoen E, Amick E, Deorari A, et al. Helping Babies Breathe, Second Edition: A Model for Strengthening Educational Programs to Increase Global Newborn Survival. Glob Health Sci Pract. 2018;538-51.

17. Trevisanuto D, Urlesberger B. European Resuscitation Council Guidelines for Resuscitation 2015: Section 7. Resuscitation and support of transition of babies at birth. Resuscitation [Internet]. 2019;95:249-63. Available from: http://dx.doi.org/10.1016/j.resuscitation.201 5.07 .029
18. WHO. WHO Technical specifications of Neonatal Resuscitation Devices. 2016;

19. WHO. World health statistics. 2018.

20. Mukhtar-yola M, Audu LI, Olaniyan O, Akinbi HT, Dawodu A, Donovan EF. Decreasing birth asphyxia : utility of statistical process control in a low- resource setting. BMJ Open Qual. 2018;

21. Simen-kapeu A, Seale AC, Wall S, Nyange C, Qazi SA, Moxon SG, et al. Treatment of neonatal infections: a multi-country analysis of health system bottlenecks and potential solutions. BMC Pregnancy Childbirth. 2015;15(Suppl 2):1-15.

22. Reisman J, Arlington L, Jensen L, Louis H. Newborn Resuscitation Training in ResourceLimited Settings: A Systematic Literature Review. 2019;138(2).

23. Hategeka C, Mwai L, Tuyisenge L. Implementing the Emergency Triage, Assessment and Treatment plus admission care ( ETAT + ) clinical practice guidelines to improve quality of hospital care in Rwandan district hospitals : healthcare workers' perspectives on relevance and challenges. Pediatrics. 2017;1-12.

24. Trevisanuto D, Cavallin F, Arnolda G, Chien TD, Lincetto O, Xuan NM, et al. Equipment for neonatal resuscitation in a middle-income country: a national survey in Vietnam. BMC Pediatr [Internet]. 2016; Available from: http:/ /dx.doi.org/10.1186/s12887-016-06640

25. WHO/MCSP. Highlights from the World Health Organization 2012 Guidelines. 2016;(June).

26. Makene CL, Plotkin M, Currie S, Bishanga D, Ugwi $\mathrm{P}$, Louis $\mathrm{H}$, et al. Improvements in newborn care and newborn resuscitation following a quality improvement program at scale: results from a before and after study in Tanzania. BMC Pregnancy and Childbirth. 2014:1-11. 